This item was submitted to Loughborough's Research Repository by the author.

Items in Figshare are protected by copyright, with all rights reserved, unless otherwise indicated.

\title{
Brittle interfacial cracking between two dissimilar elastic layers: part 1- analytical development
}

\section{PLEASE CITE THE PUBLISHED VERSION}

http://dx.doi.org/10.1016/j.compstruct.2015.06.080

\section{PUBLISHER}

(C) Elsevier

VERSION

AM (Accepted Manuscript)

\section{PUBLISHER STATEMENT}

This work is made available according to the conditions of the Creative Commons Attribution-NonCommercialNoDerivatives 4.0 International (CC BY-NC-ND 4.0) licence. Full details of this licence are available at: https://creativecommons.org/licenses/by-nc-nd/4.0/

\section{LICENCE}

CC BY-NC-ND 4.0

\section{REPOSITORY RECORD}

Harvey, Christopher, Joe Wood, and Simon Wang. 2015. "Brittle Interfacial Cracking Between Two Dissimilar Elastic Layers: Part 1-analytical Development”. Loughborough University. https://hdl.handle.net/2134/18651. 


\title{
Brittle interfacial cracking between two dissimilar elastic layers: Part 1-Analytical development
}

\author{
C. M. Harvey, J. D. Wood, S. Wang* \\ Department of Aeronautical and Automotive Engineering, Loughborough University, \\ Loughborough, Leicestershire LE11 3TU, United Kingdom
}

\begin{abstract}
Fracture on bimaterial interfaces is an important consideration in the design and application of composite materials and structures. It has, however, proved an extremely challenging problem for many decades to obtain an analytical solution for the complex stress intensity factors (SIFs) and the crack extension size-dependent energy release rates (ERRs), based on 2D elasticity. This work reports such an analytical solution for brittle interfacial cracking between two dissimilar elastic layers. The solution is achieved by developing two types of pure fracture modes and two powerful mathematical techniques. The two types of pure fracture modes are a SIF type and a load type. The two mathematical techniques are a shifting technique and an orthogonal pure mode technique. Overall, excellent agreement is observed between the analytical solutions and numerical simulations by using the finite element method (FEM). This paper reports the analytical development of the work. The numerical verification using the FEM is reported in Part 2 by Harvey, Wood and Wang (2015).
\end{abstract}

Keywords: Bimaterials, Energy release rate, Interfacial fracture, Stress intensity factors, Mixedmode partition, Orthogonal pure modes

* Corresponding Author 


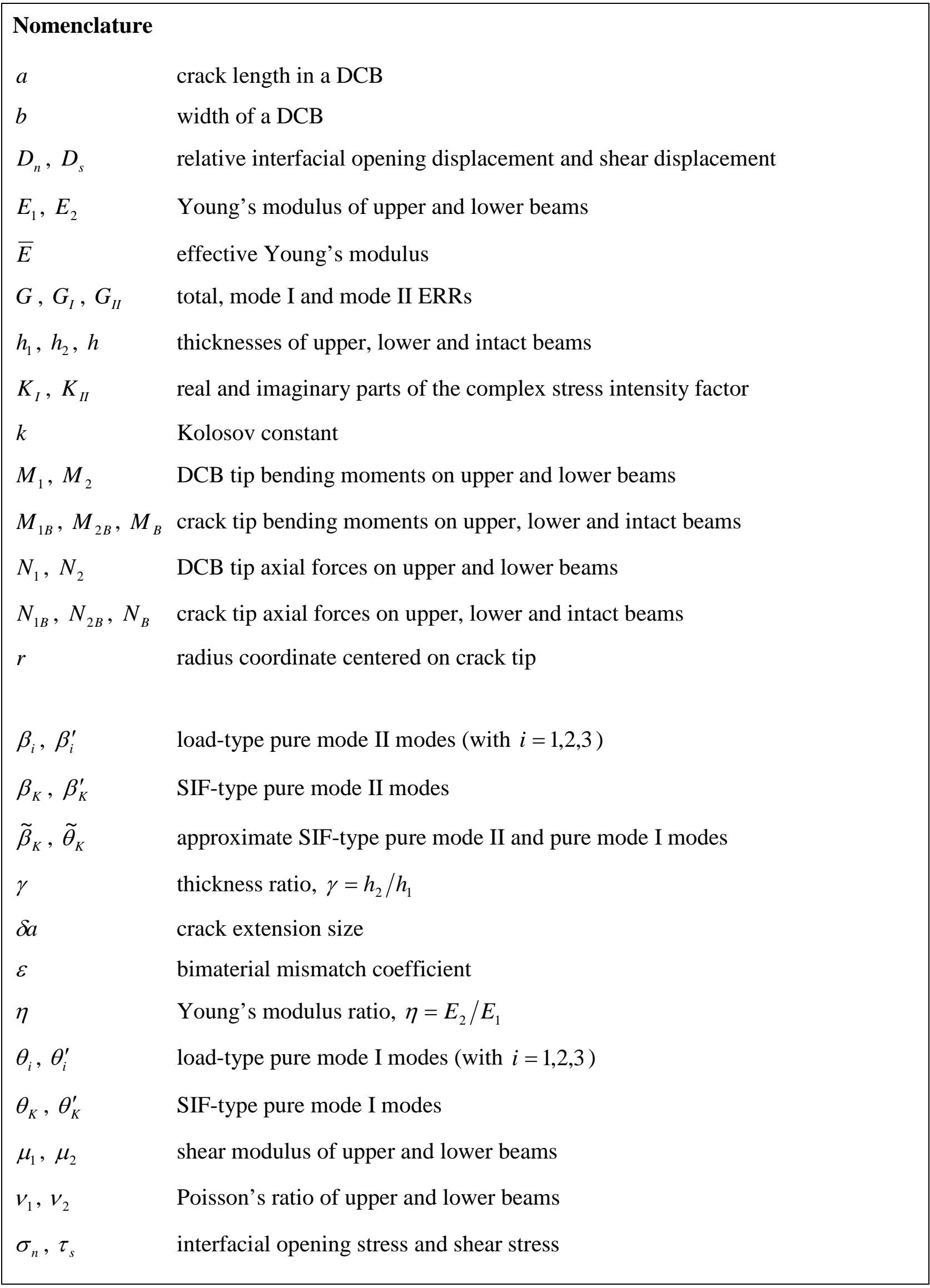




\begin{tabular}{|ll|}
\hline DCB & double cantilever beam \\
ERR & energy release rate \\
FEM & finite element method \\
SIF & stress intensity factor \\
VCCT & virtual crack closure technique \\
\hline
\end{tabular}

\section{Introduction}

Bimaterials are commonly found in both natural and artificial products. Examples include skin and tissue in biological bodies, painted metal in vehicle bodies, and thermal barrier coatings in gas turbine engines, among many others. Fracture on bimaterial interfaces remains a very important and challenging mechanics problem today.

Williams' pioneering work [1] discovered the oscillatory singularities in the elastic field around the crack tip on a bimaterial interface. Williams' work [1] is usually called the oscillatory model. Subsequently, work by Erdogan [2,3] and Rice and Sih [4] verified the presence of these oscillatory singularities; however, England's work [5] showed that the oscillation is physically inadmissible since it causes the upper and lower surfaces of the crack to wrinkle and overlap near to the crack tip. Then, in a series of work by Comninou [6-8], the inadmissible oscillatory singularities and the ensuing material interpenetration were eliminated by assuming a small frictionless contact zone near the crack tip. Comninou's work [6-8] is therefore usually called the contact model. Based on the contact model [6-8], Gautesen and Dundurs [9,10] and Gautesen [11] developed analytical theories for calculating physical quantities such as the contact zone size and the interface tractions.

It is now still an open question as to which model more closely represents the reality. Ref. [12] gives some detailed comparisons of near crack tip stress, contact zone size and oscillation zone size between the two models. Sun and Qian [12] and Rice [13] argue that the oscillatory model [1] does capture the essential stress state near the crack tip when the contact zone size is much smaller than the crack length. This may partially explain why the oscillatory model [1] appears to be more commonly accepted among researchers.

The oscillatory model, however, results in a complex stress intensity factor (SIF), various forms of which are given in Refs. [4,12,14]. This complex SIF gives rise to fundamental differences between cracks on bimaterial interfaces and interfacial cracks between similar materials (which possess a real SIF), and presents two major challenges that must be solved in order to obtain analytical solutions for $K_{I}$ and $K_{I I}$ : (1) In the case of interfacial cracks between 
similar materials, the energy release rates (ERRs), $G_{I}$ and $G_{I I}$, are each related to the corresponding SIF, $K_{I}$ or $K_{I I}$; however, in the case of cracks on bimaterial interfaces, $G_{I}$ and $G_{I I}$ are each coupled with both $K_{I}$ and $K_{I I}$ together. The first challenge is to reveal the mechanical meaning of this coupling. (2) In the case of interfacial cracks between similar materials, both the total ERR $G$ and its partition into $G_{I}$ and $G_{I I}$ are independent of the crack extension size or the finite element method (FEM) mesh size; however, in the case of cracks on bimaterial interfaces, the individual ERRs, $G_{I}$ and $G_{I I}$, vary with crack extension size or FEM mesh size, although the total ERR $G$ remains constant. The second challenge is to accurately determine $G_{I}$ and $G_{I I}$ analytically for a certain crack extension size.

These two challenges have been preventing researchers from obtaining analytical solutions for $G_{I}, G_{I I}, K_{I}$ and $K_{I I}$ for decades. Refs. [12,15-19] use numerical methods to find $G_{I}$ and $G_{I I}$. Ref. [20] relies on inconvenient discrete parameters to calculate $K_{I}$ and $K_{I I}$ that have been determined numerically. It is still widely used to study bimaterial interfacial fractures [21-23] because of the lack of better alternatives. Also, some studies simply ignore the material mismatch altogether in order to avoid using these inconvenient discrete parameters. An improvement over the discrete parameters in Ref. [20] has been made in Ref. [24] by using continuous parameter curves obtained by interpolating FEM results. The applicability of these curves is, however, limited by the range of thickness ratios and restricted loading conditions.

This work aims to present a complete analytical solution to the problem. To address the first of the challenges mentioned above, the coupling between the ERRs, $G_{I}$ and $G_{I I}$, and the SIFs, $K_{I}$ and $K_{I I}$, is studied by using the authors' orthogonal pure mode methodology [25-34] and the fundamental mechanical meaning of the coupling is revealed. The second challenge is then overcome by using two powerful mathematical techniques: The first technique is developed in this work and is called the shifting technique; the second technique again makes use of the authors' orthogonal pure mode methodology [25-34]. Accurate analytical solutions are achieved for the crack extension size-independent SIFs, $K_{I}$ and $K_{I I}$, and the crack extension sizedependent ERRs, $G_{I}$ and $G_{I I}$. The work is reported in two parts: first, the analytical development, and second, the numerical verification. This paper contains the Part 1 . Part 2 is reported in Ref. [35].

Before presenting the work, it may be useful to give the following notes. Recently, Wang and Harvey and their colleagues [25-34] have developed analytical mixed-mode partition theories for one-dimensional delamination in laminated composite beams and plates by using a powerful 
methodology, which is fundamentally different from the conventional ones. It has been shown that Wang and Harvey's Euler beam mixed-mode partition theory gives excellent fracture toughness predictions for delamination in generally laminated composite beams [29,34] by using thorough and comprehensive experimental test data [36-43]. In contrast, the 2D elasticity-based mixed-mode partition theory [33,44] gives poor predictions. The very latest study [45] shows that for buckling driven delamination, the Euler beam mixed-mode partition theory also gives more accurate predictions of fracture toughness than the 2D elasticity-based partition theory does. This is because the brittle fracture propagation is governed by global ERR partitions. Readers are referred to Refs. [29,34] for detailed explanations. The finite fracture mechanics approach [46] is also helpful and complements the explanations. It is still unknown, however, whether the Euler beam mixed-mode partition theory governs brittle fracture in other loading conditions such as fatigue loading, thermal loading, etc. It is therefore still very necessary to develop mixed-mode partition theories based on 2D elasticity in order to provide a complete set of tools for the study of interfacial fractures between dissimilar materials and this is the motivation of the present work.

\section{Analytical development}

\subsection{Interfacial stresses ahead of the crack tip}

Fig. 1a shows a bimaterial double cantilever beam (DCB) with its material properties, geometry and loading conditions. The Young's modulus, shear modulus and Poisson's ratio of beam $i$ are denoted by $E_{i}, \mu_{i}$, and $v_{i}$ respectively (with $i=1,2$ ). The interfacial opening stress and shear stress ahead of the crack tip, $\sigma_{n}$ and $\tau_{s}$, can be expressed in a combined complex form as [20]

$$
\sigma_{n}+i \tau_{s}=\frac{\left(K_{I}+i K_{I I}\right)}{\sqrt{2 \pi r}} r^{i \varepsilon}
$$

or in individual real form as

$$
\begin{aligned}
& \sigma_{n}=\frac{1}{\sqrt{2 \pi r}}\left\{K_{I} \cos [\varepsilon \ln (r)]-K_{I I} \sin [\varepsilon \ln (r)]\right\} \\
& \tau_{s}=\frac{1}{\sqrt{2 \pi r}}\left\{K_{I} \sin [\varepsilon \ln (r)]+K_{I I} \cos [\varepsilon \ln (r)]\right\}
\end{aligned}
$$


where $r$ is the radius coordinate centered on crack tip. The signs of $\sigma_{n}$ and $\tau_{s}$ are positive in the directions shown in Fig. 1b. In Eqs. (1), (2) and (3), the bimaterial constant $\varepsilon$ is defined as

$$
\varepsilon=\frac{1}{2 \pi} \ln \left[\left(\frac{k_{1}}{\mu_{1}}+\frac{1}{\mu_{2}}\right)\left(\frac{k_{2}}{\mu_{2}}+\frac{1}{\mu_{1}}\right)^{-1}\right]
$$

where the Kolosov constant $k_{i}$ (with $i=1,2$ ) is defined as $k_{i}=3-4 v_{i}$ for plane strain and $k_{i}=\left(3-v_{i}\right) /\left(1+v_{i}\right)$ for plane stress. It is easy to verify that when $v_{1}=v_{2}$ then $\varepsilon\left(\eta^{-1}\right)=-\varepsilon(\eta)$ where $\eta=E_{2} / E_{1}$ is the Young's modulus ratio.

\subsection{Relative interfacial displacements behind the crack tip}

Based on Refs. [12,15], the relative interfacial opening displacement behind the crack tip $D_{n}$ and the relative interfacial shear displacement behind the crack tip $D_{s}$, of the upper beam 1 with respect to the lower beam 2, can be expressed in individual real form as

$$
\begin{aligned}
D_{n} & =D \cos (\xi) \sqrt{2 \pi r}\left\{K_{I} \cos [\varepsilon \ln (r)-\xi]-K_{I I} \sin [\varepsilon \ln (r)-\xi]\right\} \\
& =D \cos (\xi) \sqrt{2 \pi r} \cos [\varepsilon \ln (r)-\xi]\left(K_{I}-\widetilde{\beta}_{K}{ }^{-1} K_{I I}\right) \\
D_{s} & =D \cos (\xi) \sqrt{2 \pi r}\left\{K_{I} \sin [\varepsilon \ln (r)-\xi]+K_{I I} \cos [\varepsilon \ln (r)-\xi]\right\} \\
& =D \cos (\xi) \sqrt{2 \pi r} \sin [\varepsilon \ln (r)-\xi]\left(K_{I}-\tilde{\theta}_{K}^{-1} K_{I I}\right)
\end{aligned}
$$

where the signs of $D_{n}$ and $D_{s}$ are consistent with the signs of the interfacial stresses shown in Fig. 1b, and

$$
\begin{gathered}
D=\frac{\left(C_{1}+C_{2}\right)}{4 \pi \cosh (\pi \varepsilon)} \\
\tilde{\theta}_{K}=-\tan [\varepsilon \ln (r)-\xi] \quad \tilde{\beta}_{K}=\frac{1}{\tan [\varepsilon \ln (r)-\xi]}
\end{gathered}
$$

with

$$
\begin{gathered}
C_{i}=\frac{1+k_{i}}{\mu_{i}} \quad \text { with } \quad i=1,2 \\
\cos (\xi)=\frac{1}{\left(1+4 \varepsilon^{2}\right)^{1 / 2}} \quad \sin (\xi)=\frac{2 \varepsilon}{\left(1+4 \varepsilon^{2}\right)^{1 / 2}}
\end{gathered}
$$


Note that $\tilde{\theta}_{K} \tilde{\beta}_{K}=-1$ due to pure mode orthogonality which is discussed later in Section 2.3. Also note that $\tilde{\theta}_{K}(-\varepsilon)=-\tilde{\theta}_{K}(\varepsilon)$ and $\tilde{\beta}_{K}(-\varepsilon)=-\widetilde{\beta}_{K}(\varepsilon)$ due to physical symmetry, which become $\tilde{\theta}_{K}\left(\eta^{-1}\right)=-\tilde{\theta}_{K}(\eta)$ and $\tilde{\beta}_{K}\left(\eta^{-1}\right)=-\widetilde{\beta}_{K}(\eta)$ when $v_{1}=v_{2}$. It is seen from Eqs. (2), (3), (5) and (6) that the interfacial stresses and the relative interfacial displacements are out of phase by $\xi$ because of the bimaterial mismatch constant $\varepsilon$. The profound mechanical meaning of this phase difference will be shown in next section for ERR partitions.

2.3. Partitioning the ERR G using pure modes in terms of $K_{I}$ and $K_{I I}$

The relationships between the ERRs, $G_{I}$ and $G_{I I}$, and the SIFs, $K_{I}$ and $K_{I I}$, are traditionally obtained by using the virtual crack closure technique (VCCT). The following relationships were originally derived in Refs. [12,15] using the VCCT, but they are written here in a different form and also use the SIFs in Eq. (1) [20] for more convenient calculations:

$$
\begin{gathered}
G=G_{I}+G_{I I}=\frac{D \pi}{4 \cosh (\pi \varepsilon)}\left(K_{I}{ }^{2}+K_{I I}{ }^{2}\right) \\
G_{d}=G_{I}-G_{I I}=D|B| \cos ^{2}(\xi)\left(1+\frac{\delta a}{2 a}\right)\left[\left(K_{I}{ }^{2}-K_{I I}{ }^{2}\right) \cos (\bar{\rho})+2 K_{I} K_{I I} \sin (\bar{\rho})\right]
\end{gathered}
$$

The individual ERRs, $G_{I}$ and $G_{I I}$, can then be written as

$$
\begin{aligned}
& G_{I}=\frac{G}{2}+\frac{D|B| \cos ^{2}(\xi)}{2}\left(1+\frac{\delta a}{2 a}\right)\left[\left(K_{I}{ }^{2}-K_{I I}{ }^{2}\right) \cos (\bar{\rho})+2 K_{I} K_{I I} \sin (\bar{\rho})\right] \\
& G_{I I}=\frac{G}{2}-\frac{D|B| \cos ^{2}(\xi)}{2}\left(1+\frac{\delta a}{2 a}\right)\left[\left(K_{I}{ }^{2}-K_{I I}{ }^{2}\right) \cos (\bar{\rho})+2 K_{I} K_{I I} \sin (\bar{\rho})\right]
\end{aligned}
$$

where

$$
\begin{gathered}
B=0.5 \pi^{0.5}(0.5+i \varepsilon) \frac{\Gamma(0.5-i \varepsilon)}{\Gamma(1-i \varepsilon)}=B_{\mathrm{Re}}+i B_{\mathrm{Im}} \\
\bar{\rho}=\rho-2 \varepsilon \ln \left(\frac{\delta a}{2}\right) \\
\cos (\rho)=\frac{B_{\mathrm{Re}}}{|B|} \quad \sin (\rho)=\frac{B_{\mathrm{Im}}}{|B|}
\end{gathered}
$$


The notation $|B|$ represents the complex modulus of $B$. The gamma function is represented by $\Gamma$, and the crack extension size by $\delta a$. Eq. (11) shows that the total ERR $G$ is independent of $\delta a$, but Eqs. (12) to (14) show that the individual ERRs, $G_{I}$ and $G_{I I}$, are dependent on $\delta a$. By solving Eqs. (11) and (12) together, $K_{I}^{2}$ can be expressed as

$$
K_{I}^{2}=\frac{2 \cosh (\pi \varepsilon)}{D \pi}\left[G+\cos (\bar{\rho}) C_{d} G_{d} \pm \sin (\bar{\rho})\left(G^{2}-C_{d}^{2} G_{d}^{2}\right)^{1 / 2}\right]
$$

where

$$
C_{d}=\frac{\pi}{4|B| \cosh (\pi \varepsilon) \cos ^{2}(\xi)[1+\delta a /(2 a)]}
$$

Note that $C_{d}$ is close to 1 . Eq. (18) gives two values for $K_{I}^{2}$ if $\varepsilon \neq 0$, denoted here by $\left(K_{I}^{2}\right)_{1}$ and $\left(K_{I}^{2}\right)_{2}$. The corresponding two values of $K_{I I}{ }^{2}$ are $\left(K_{I I}{ }^{2}\right)_{1}$ and $\left(K_{I I}{ }^{2}\right)_{2}$ respectively and they can be obtained by using Eq. (11). The four pairs of solutions for $K_{I}$ and $K_{I I}$, denoted by $K_{I-1}$ to $K_{I-4}$ and $K_{I I-1}$ to $K_{I I-4}$ respectively, can be found by using Eq. (12). From Eq. (12), we have

$$
K_{I} K_{I I}=\frac{G_{d}}{2 D|B| \cos ^{2}(\xi) \sin (\bar{\rho})[1+\delta a /(2 a)]}-\frac{\left(K_{I}{ }^{2}-K_{I I}{ }^{2}\right)}{2 \tan (\bar{\rho})}
$$

which has two values, $\left(K_{I} K_{I I}\right)_{1}$ and $\left(K_{I} K_{I I}\right)_{2}$, based on the two pairs of $K_{I}{ }^{2}$ and $K_{I I}{ }^{2}$. If $\left(K_{I} K_{I I}\right)_{1}>0$ then

$$
K_{I-1}=+\sqrt{\left(K_{I}^{2}\right)_{1}} \quad K_{I I-1}=+\sqrt{\left(K_{I I}^{2}\right)_{1}} \quad K_{I-2}=-\sqrt{\left(K_{I}^{2}\right)_{1}} \quad K_{I I-2}=-\sqrt{\left(K_{I I}^{2}\right)_{1}}
$$

Otherwise if $\left(K_{I} K_{I I}\right)_{1}<0$

$$
K_{I-1}=+\sqrt{\left(K_{I}^{2}\right)_{1}} \quad K_{I I-1}=-\sqrt{\left(K_{I I}^{2}\right)_{1}} \quad K_{I-2}=-\sqrt{\left(K_{I}^{2}\right)_{1}} \quad K_{I I-2}=+\sqrt{\left(K_{I I}^{2}\right)_{1}}
$$

Similarly, if $\left(K_{I} K_{I I}\right)_{2}>0$ then

$$
K_{I-3}=+\sqrt{\left(K_{I}^{2}\right)_{2}} \quad K_{I I-3}=+\sqrt{\left(K_{I I}^{2}\right)_{2}} \quad K_{I-4}=-\sqrt{\left(K_{I}^{2}\right)_{2}} \quad K_{I I-4}=-\sqrt{\left(K_{I I}^{2}\right)_{2}}
$$

otherwise if $\left(K_{I} K_{I I}\right)_{2}<0$

$$
K_{I-3}=+\sqrt{\left(K_{I}^{2}\right)_{2}} \quad K_{I I-3}=-\sqrt{\left(K_{I I}^{2}\right)_{2}} \quad K_{I-4}=-\sqrt{\left(K_{I}^{2}\right)_{2}} \quad K_{I I-4}=+\sqrt{\left(K_{I I}^{2}\right)_{2}}
$$


Note that if $\varepsilon=0$ then $\bar{\rho}=0$ and the four pairs of solutions for $K_{I}$ and $K_{I I}$ can be easily calculated using Eqs. (11) and (18). In all cases, however, there will only be one mechanically admissible pair for a given loading condition, from among the complete set of mathematical solutions for $K_{I}$ and $K_{I I}$. FEM simulations were used in Ref. [12] to determine this pair. In this work, a method is devised to guide the selection of the correct pair by purely analytical means.

In the case of interfacial cracks between similar materials, that is, when the material mismatch coefficient $\varepsilon=0$, the ERRs, $G_{I}$ and $G_{I I}$, are each related to the corresponding SIF, $K_{I}$ or $K_{I I}$; however, in the case of cracks on bimaterial interfaces, that is, when $\varepsilon \neq 0, G_{I}$ and $G_{I I}$ are each coupled with both $K_{I}$ and $K_{I I}$ together, as shown by Eqs. (13) and (14). What is the mechanical meaning of the coupling? In Wang and Harvey’s [25-34] previous work, a powerful orthogonal pure mode technique has been developed for partitioning mixed-mode fractures. Orthogonal pure modes are derived in terms of the applied crack tip forces and moments. Here, it is expected that pure modes also exist in terms of the SIFs, $K_{I}$ and $K_{I I}$, because SIFs can be considered as alternative form of load. Based on this mechanical understanding, and by writing the pure modes in terms of the SIFs, $K_{I}$ and $K_{I I}$, the total ERR $G$ in Eq. (11), is partitioned as

$$
\begin{gathered}
G_{I}=\frac{D \pi}{4 \cosh (\pi \varepsilon)\left(1+\beta_{K}^{-1} \beta_{K}^{\prime-1}\right)}\left(K_{I}-\beta_{K}^{-1} K_{I I}\right)\left(K_{I}-\beta_{K}^{\prime-1} K_{I I}\right) \\
G_{I I}=\frac{D \pi}{4 \cosh (\pi \varepsilon)\left(1+\theta_{K}^{-1} \theta_{K}^{\prime-1}\right)}\left(K_{I}-\theta_{K}^{-1} K_{I I}\right)\left(K_{I}-\theta_{K}^{\prime-1} K_{I I}\right)
\end{gathered}
$$

From Eq. (26) it is seen that when $K_{I I}=\theta_{K} K_{I}$ then $G_{I I}=0$. The relationship, $K_{I I}=\theta_{K} K_{I}$, produces a pure mode I $\theta_{K}$ fracture. Also, from Eq. (26), when $K_{I I}=\theta_{K}^{\prime} K_{I}$ then $G_{I I}=0$. The relationship $K_{I I}=\theta_{K}^{\prime} K_{I}$ produces a pure mode I $\theta_{K}^{\prime}$ fracture. The physical meanings of these two pure mode I modes are zero effective relative crack tip shear displacement and zero effective crack tip shear force respectively. When the bimaterial mismatch constant $\varepsilon \neq 0$, it is seen from Eqs. (2), (3), (5) and (6) that the variations of interfacial stresses and the relative interfacial displacements are out of phase by $\xi$. This causes $\theta_{K} \neq \theta_{K}^{\prime}$ and leads to two pure mode I modes.

Similarly, from Eq. (25) it is seen that when $K_{I I}=\beta_{K} K_{I}$ then $G_{I}=0$. The relationship, $K_{I I}=\beta_{K} K_{I}$, produces a pure mode II $\beta_{K}$ fracture. Also, from Eq. (25), when $K_{I I}=\beta_{K}^{\prime} K_{I}$ then $G_{I}=0$. The relationship $K_{I I}=\beta_{K}^{\prime} K_{I}$ produces a pure mode II $\beta_{K}^{\prime}$ fracture. The physical meanings of these two pure mode II modes are zero effective crack tip opening force and zero 
effective crack tip relative opening displacement respectively. Generally, $\beta_{K} \neq \beta_{K}^{\prime}$ and there are two pure mode II modes.

These four pure modes, $\theta_{K}, \theta_{K}^{\prime}, \beta_{K}$ and $\beta_{K}^{\prime}$, form two sets of orthogonal pure modes. The first orthogonal set is $\left(\theta_{K}, \beta_{K}\right)$, and the second orthogonal set is $\left(\theta_{K}^{\prime}, \beta_{K}^{\prime}\right)$. Taking the $\left(\theta_{K}, \beta_{K}\right)$ set as an example, here, orthogonal means that

$$
\left(\begin{array}{ll}
1 & \theta_{K}
\end{array}\right)\left[\begin{array}{ll}
1 & 0 \\
0 & 1
\end{array}\right]\left(\begin{array}{c}
1 \\
\beta_{K}
\end{array}\right)=0
$$

where the square matrix is the coefficient matrix of the total ERR $G$, given in Eq. (11). Obviously, $\theta_{K} \beta_{K}=-1$. Similarly, $\theta_{K}^{\prime} \beta_{K}^{\prime}=-1$. One important consequence of the existence of two sets of pure modes is that negative $G_{I}$ or $G_{I I}$ can occur (similarly, so can $G_{I}>G$ or $G_{I I}>G$ ) as seen from Eqs. (25) and (26). Note that the total ERR $G$ is still non-negativedefinite. The situation here is very similar to Wang and Harvey's [25-34] Euler beam partition theory for mixed-mode fractures, in which there are also two sets of pure modes, but which are caused by the 'global' nature of the partition (i.e. the Euler beam partition is equivalent to the elasticity-based partition calculated over the entire region that is mechanically affected by the crack tip). Here, however, the two sets of pure modes are caused by the out-of-phase oscillation of the relative displacements and stresses near to crack tip, which are 'local' in nature. By letting $G_{I I}=0$ and $G_{I}=0$ in Eqs. (14) and (13), respectively, the pure modes are found to be

$$
\begin{gathered}
\theta_{K}=\left\{\begin{array}{l}
{\left[\sin (\bar{\rho})+\sqrt{1-C_{d}^{2}}\right] /\left[C_{d}+\cos (\bar{\rho})\right] \text { if } \varepsilon<0} \\
{\left[\sin (\bar{\rho})-\sqrt{1-C_{d}^{2}}\right] /\left[C_{d}+\cos (\bar{\rho})\right] \text { if } \varepsilon>0}
\end{array}\right. \\
\theta_{K}^{\prime}=\left\{\begin{array}{l}
{\left[\sin (\bar{\rho})-\sqrt{1-C_{d}^{2}}\right] /\left[C_{d}+\cos (\bar{\rho})\right] \text { if } \varepsilon<0} \\
{\left[\sin (\bar{\rho})+\sqrt{1-C_{d}^{2}}\right] /\left[C_{d}+\cos (\bar{\rho})\right] \text { if } \varepsilon>0}
\end{array}\right. \\
\beta_{K}=\left\{\begin{array}{l}
{\left[-\sin (\bar{\rho})+\sqrt{1-C_{d}^{2}}\right] /\left[C_{d}+\cos (\bar{\rho})\right] \text { if } \varepsilon<0} \\
{\left[-\sin (\bar{\rho})-\sqrt{1-C_{d}^{2}}\right] /\left[C_{d}+\cos (\bar{\rho})\right] \text { if } \varepsilon>0}
\end{array}\right. \\
\beta_{K}^{\prime}=\left\{\begin{array}{l}
{\left[-\sin (\bar{\rho})-\sqrt{1-C_{d}^{2}}\right] /\left[C_{d}+\cos (\bar{\rho})\right] \text { if } \varepsilon<0} \\
{\left[-\sin (\bar{\rho})+\sqrt{1-C_{d}^{2}}\right] /\left[C_{d}+\cos (\bar{\rho})\right] \text { if } \varepsilon>0}
\end{array}\right.
\end{gathered}
$$


Note that $\theta_{K}(-\varepsilon)=-\theta_{K}(\varepsilon), \theta_{K}^{\prime}(-\varepsilon)=-\theta_{K}^{\prime}(\varepsilon), \beta_{K}(-\varepsilon)=-\beta_{K}(\varepsilon)$ and $\beta_{K}^{\prime}(-\varepsilon)=-\beta_{K}^{\prime}(\varepsilon)$ due to physical symmetry, which become $\theta_{K}(\eta)=-\theta_{K}\left(\eta^{-1}\right), \theta_{K}^{\prime}(\eta)=-\theta_{K}^{\prime}\left(\eta^{-1}\right), \beta_{K}\left(\eta^{-1}\right)=-\beta_{K}(\eta)$ and $\beta_{K}^{\prime}\left(\eta^{-1}\right)=-\beta_{K}^{\prime}(\eta)$ when $v_{1}=v_{2}$.

It is now clear that the mechanical meaning of the coupling between the ERRs, $G_{I}$ and $G_{I I}$, and the SIFs, $K_{I}$ and $K_{I I}$, is the existence of two sets of orthogonal pure modes, $\left(\theta_{K}, \beta_{K}\right)$ and $\left(\theta_{K}^{\prime}, \beta_{K}^{\prime}\right)$. Based on this understanding and the partition given by Eqs. (25) and (26), a method is now devised to guide the selection of the mechanically admissible SIF pair, $K_{I}$ and $K_{I I}$ by purely analytical means. The idea comes from the fact that when the bimaterial mismatch constant $\varepsilon$ is not large, the two pure mode I modes, $\theta_{K}$ and $\theta_{K}^{\prime}$, are close to each other. This is also true for the two pure mode II modes, $\beta_{K}$ and $\beta_{K}^{\prime}$. It is therefore reasonable to expect that middle values between these two pure modes are good approximations. Using Eq. (6) and the condition $D_{s}(\delta a)=0$ gives the approximate pure mode I condition relationship,

$$
K_{I I}=\tilde{\theta}_{K} K_{I}
$$

The variation of $\theta_{K}, \theta_{K}^{\prime}$ and $\tilde{\theta}_{K}$ with respect to $\delta a$ is shown in Fig. 2 for different values of the bimaterial mismatch constant $\varepsilon$. As expected, $\tilde{\theta}_{K}$ is close to $\theta_{K}$ for all values of $\delta a$ and $\varepsilon$, and is between $\theta_{K}$ and $\theta_{K}^{\prime}$, which demonstrates that $\tilde{\theta}_{K}$ is a good approximation.

Similarly, using Eq. (5) and the condition $D_{n}(\delta a)=0$ gives the approximate pure mode II relationship,

$$
K_{I I}=\widetilde{\beta}_{K}^{\prime} K_{I}
$$

Note that in Eq. (33), $\tilde{\beta}_{K}^{\prime}$ is used in place of the $\tilde{\beta}_{K}$ in Eq. (5) for consistency with the discussion above regarding Eqs. (25) and (26), where $\theta_{K}$ and $\beta_{K}^{\prime}$ give the values of $K_{I I} / K_{I}$ required for zero effective relative displacements. Here $\tilde{\theta}_{K}$ and $\tilde{\beta}_{K}^{\prime}$ give the values of $K_{I I} / K_{I}$ required for zero relative displacements at $x=\delta a$. It is easy to show that $\tilde{\theta}_{K}$ and $\tilde{\beta}_{K}^{\prime}$ are orthogonal to each other, that is,

$$
\left(\begin{array}{ll}
1 & \tilde{\theta}_{K}
\end{array}\right)\left[\begin{array}{ll}
1 & 0 \\
0 & 1
\end{array}\right]\left(\begin{array}{c}
1 \\
\tilde{\beta}_{K}^{\prime}
\end{array}\right)=0
$$


Physically though, $\tilde{\theta}_{K}$ is orthogonal to $\widetilde{\beta}_{K}$, as indicated by Eq. (27) and also as explained in Refs. [25-34]. Therefore Eq. (34) implies that $\tilde{\beta}_{K}^{\prime}=\tilde{\beta}_{K}$ and consequently that $\tilde{\theta}_{K}^{\prime}=\tilde{\theta}_{K}$. It is easy to validate this from Eq. (1) by showing that $F_{n}(\delta a)=\int_{0}^{\delta a} \sigma_{n} d r=0$ when $D_{n}(\delta a)=0$ and that $F_{s}(\delta a)=\int_{0}^{\delta a} \tau_{s} d r=0$ when $D_{s}(\delta a)=0$. Therefore, the approximate pure mode sets, $\left(\tilde{\theta}_{K}, \tilde{\beta}_{K}\right)$ and $\left(\tilde{\theta}_{K}^{\prime}, \tilde{\beta}_{K}^{\prime}\right)$, coincide with each other and $\tilde{\theta}_{K} \tilde{\beta}_{K}=-1$ from Eq. (34). An approximate partition is then obtained as

$$
\begin{aligned}
& G_{I}=\frac{D \pi}{4 \cosh (\pi \varepsilon)\left(1+\tilde{\beta}_{K}^{-2}\right)}\left(K_{I}-\tilde{\beta}_{K}^{-1} K_{I I}\right)^{2} \\
& G_{I I}=\frac{D \pi}{4 \cosh (\pi \varepsilon)\left(1+\tilde{\theta}_{K}^{-2}\right)}\left(K_{I}-\tilde{\theta}_{K}^{-1} K_{I I}\right)^{2}
\end{aligned}
$$

From Eq. (35),

$$
K_{I}-\widetilde{\beta}_{K}^{-1} K_{I I}= \pm \sqrt{\bar{G}_{I}} \sqrt{1+\widetilde{\beta}_{K}^{-2}}
$$

where

$$
\bar{G}_{I}=\frac{4 \cosh (\pi \varepsilon) G_{I}}{D \pi}
$$

Eq. (5) shows that $\operatorname{sgn}\left(K_{I}-\widetilde{\beta}_{K}{ }^{-1} K_{I I}\right)=\operatorname{sgn}\left[D_{n} / \cos (\varepsilon \ln (\delta a)-\xi)\right] \equiv S_{n}$. That is,

$$
K_{I}-\widetilde{\beta}_{K}^{-1} K_{I I}=S_{n} \sqrt{\bar{G}_{I}} \sqrt{1+\widetilde{\beta}_{K}^{-2}}
$$

It is seen that as long as the sign of $D_{n}$ is known, then the sign of $\left(K_{I}-\widetilde{\beta}_{K}{ }^{-1} K_{I I}\right)$ in Eq. (37) is also known. Note that $\operatorname{sgn}\left(D_{n}\right)$ will be determined in the next section. Similarly, from Eq. (36),

$$
K_{I}-\tilde{\theta}_{K}^{-1} K_{I I}= \pm \sqrt{\bar{G}_{I I}} \sqrt{1+\widetilde{\theta}_{K}^{-2}}
$$

where

$$
\bar{G}_{I I}=\frac{4 \cosh (\pi \varepsilon) G_{I I}}{D \pi}
$$

Eq. (6) shows that $\operatorname{sgn}\left(K_{I}-\tilde{\theta}_{K}^{-1} K_{I I}\right)=\operatorname{sgn}\left[D_{s} / \sin (\varepsilon \ln (\delta a)-\xi)\right] \equiv S_{s}$. That is,

$$
K_{I}-\tilde{\theta}_{K}^{-1} K_{I I}=S_{s} \sqrt{\bar{G}_{I I}} \sqrt{1+\tilde{\theta}_{K}^{-2}}
$$


It is seen that as long as the sign of $D_{s}$ is known, then the sign of $\left(K_{I}-\tilde{\theta}_{K}{ }^{-1} K_{I I}\right)$ in Eq. (40) is known. Note that $\operatorname{sgn}\left(D_{s}\right)$ will be determined in next section. Finally, when the ERRs, $G_{I}$ and $G_{I I}$, are known, a unique pair of approximate SIFs, $K_{I}$ and $K_{I I}$, can be determined from Eqs. (39) and (42), as follows:

$$
\begin{gathered}
K_{I}= \begin{cases}\left(S_{n} \sqrt{\bar{G}_{I}} \sqrt{1+\tilde{\theta}_{K}{ }^{2}}+\tilde{\theta}_{K}{ }^{2} S_{s} \sqrt{\bar{G}_{I I}} \sqrt{1+\tilde{\beta}_{K}{ }^{2}}\right) /\left(1+\tilde{\theta}_{K}{ }^{2}\right) & \text { if } \tilde{\theta}_{K} \neq 0 \text { and } \tilde{\beta}_{K} \neq 0 \\
S_{s} \sqrt{\bar{G}_{I I}} & \text { if } \tilde{\theta}_{K}=0\end{cases} \\
K_{I I}= \begin{cases}\left(S_{n} \sqrt{\bar{G}_{I}} \sqrt{1+\tilde{\theta}_{K}^{2}}-S_{s} \sqrt{\bar{G}_{I I}} \sqrt{1+\tilde{\beta}_{K}^{2}}\right) /\left(\tilde{\theta}_{K}-\tilde{\beta}_{K}\right) & \text { if } \tilde{\beta}_{K}=0 \\
-S_{s} \sqrt{\bar{G}_{I I}} & \text { if } \tilde{\theta}_{K} \neq 0 \text { and } \tilde{\beta}_{K} \neq 0 \\
-S_{n} \sqrt{\bar{G}_{I}} & \text { if } \tilde{\beta}_{K}=0\end{cases}
\end{gathered}
$$

This approximate pair of SIFs, $K_{I}$ and $K_{I I}$, from Eqs. (43) and (44), should be used to guide the choice of the one admissible pair of SIFs, $K_{I}$ and $K_{I I}$, from Eqs. (21) to (24).

It can now be concluded that the first challenge, stated in the Introduction, has been overcome. Now, in the following development, the aim is to overcome the second challenge.

\subsection{Partitioning the ERR G using pure modes in terms of the crack tip loads}

As seen earlier, the SIFs for rigid bimaterial interfaces being complex implies that the interfacial stresses ahead of the crack tip are out of phase with the relative interfacial displacements behind the crack tip. Therefore, two sets of orthogonal pure modes must exist. Based on the authors’ previous work [25-34], the ERR partitions must be in the form,

$$
\begin{aligned}
G_{I} & =c_{I}\left(M_{1 B}-\frac{M_{2 B}}{\beta_{1}}-\frac{N_{1 B}}{\beta_{2}}-\frac{N_{2 B}}{\beta_{3}}\right)\left(M_{1 B}-\frac{M_{2 B}}{\beta_{1}^{\prime}}-\frac{N_{1 B}}{\beta_{2}^{\prime}}-\frac{N_{2 B}}{\beta_{3}^{\prime}}\right) \\
G_{I I} & =c_{I I}\left(M_{1 B}-\frac{M_{2 B}}{\theta_{1}}-\frac{N_{1 B}}{\theta_{2}}-\frac{N_{2 B}}{\theta_{3}}\right)\left(M_{1 B}-\frac{M_{2 B}}{\theta_{1}^{\prime}}-\frac{N_{1 B}}{\theta_{2}^{\prime}}-\frac{N_{2 B}}{\theta_{3}^{\prime}}\right)
\end{aligned}
$$

where $c_{I}$ and $c_{I I}$ are given in Appendix $\mathrm{A}$, and $\left(\theta_{i}, \beta_{i}\right)$ and $\left(\theta_{i}^{\prime}, \beta_{i}^{\prime}\right)$ (with $i=1,2,3$ ) represent the two sets of orthogonal pure modes. Note that both sets of modes depend on the crack extension size $\delta a$. Fig. 3 shows the variation of $\theta_{1}$ and $\theta_{1}^{\prime}$, as determined from 2D FEM 
simulations using interfacial point springs $[47,48,49]$, with respect to $\delta a$ for different values of the bimaterial mismatch constant $\varepsilon$. Three stages are seen. In the first stage, $\delta a$ is large and $\theta_{1}$ and $\theta_{1}^{\prime}$ are separated from each other for all the values of $\varepsilon$. This is caused by the global nature of the partition when $\delta a$ is large. This behavior is described by Wang and Harvey's Euler beam partition theory for mixed-mode fractures [25-34]. In the second stage, $\delta a$ is small and $\theta_{1}$ and $\theta_{1}^{\prime}$ approach to each other for all the values of $\varepsilon$. This is due to the diminishing global nature of the partition as $\delta a$ reduces in size. In all cases, $\theta_{1}$ and $\theta_{1}^{\prime}$ are coincident at approximately $\delta a=0.05$. Note from Fig. 2 that the coincidence of $\theta_{1}$ and $\theta_{1}^{\prime}$ does not result in the coincidence of $\theta_{K}$ and $\theta_{K}^{\prime}$. It is also worth noting that after the coincidence $\theta_{1}$ and $\theta_{1}^{\prime}$, (1) for $\varepsilon=0$ they remain coincident and converged, and (2) for $\varepsilon \neq 0$ they remain coincident for a certain range of $\delta a$ but not converged. In the third stage, $\delta a$ is extremely small and $\theta_{1}$ and $\theta_{1}^{\prime}$ remain converged for the case where $\varepsilon=0$ but otherwise diverge away from each other. This is due to the oscillation of the interfacial stresses and relative interfacial displacements near to the crack tip. In the second stage, where the two sets of pure modes, $\left(\theta_{i}, \beta_{i}\right)$ and $\left(\theta_{i}^{\prime}, \beta_{i}^{\prime}\right)$, coincide with each other, the partitions become

$$
\begin{aligned}
& G_{I}=c_{I}\left(M_{1 B}-\frac{M_{2 B}}{\beta_{1}}-\frac{N_{1 B}}{\beta_{2}}-\frac{N_{2 B}}{\beta_{3}}\right)^{2} \\
& G_{I I}=c_{I I}\left(M_{1 B}-\frac{M_{2 B}}{\theta_{1}}-\frac{N_{1 B}}{\theta_{2}}-\frac{N_{2 B}}{\theta_{3}}\right)^{2}
\end{aligned}
$$

from which the signs of the relative interfacial opening and shear displacements, $D_{n}$ and $D_{s}$, are then obtained as

$$
\begin{aligned}
& \operatorname{sgn}\left(D_{n}\right)= \begin{cases}-\operatorname{sgn}\left(M_{1 B}-\frac{M_{2 B}}{\beta_{1}}-\frac{N_{1 B}}{\beta_{2}}-\frac{N_{2 B}}{\beta_{3}}\right) & \text { if } \beta_{1}<0 \text { and } \eta>1 \\
+\operatorname{sgn}\left(M_{1 B}-\frac{M_{2 B}}{\beta_{1}}-\frac{N_{1 B}}{\beta_{2}}-\frac{N_{2 B}}{\beta_{3}}\right) & \text { otherwise }\end{cases} \\
& \operatorname{sgn}\left(D_{s}\right)= \begin{cases}+\operatorname{sgn}\left(M_{1 B}-\frac{M_{2 B}}{\theta_{1}}-\frac{N_{1 B}}{\theta_{2}}-\frac{N_{2 B}}{\theta_{3}}\right) & \text { if } \theta_{1}>0 \text { and } \eta<1 \\
-\operatorname{sgn}\left(M_{1 B}-\frac{M_{2 B}}{\theta_{1}}-\frac{N_{1 B}}{\theta_{2}}-\frac{N_{2 B}}{\theta_{3}}\right) & \text { otherwise }\end{cases}
\end{aligned}
$$


Note that in most cases, $\theta_{1}$ and $\beta_{1}$ are negative and positive respectively, in which the second solutions in Eqs. (49) and (50) are the correct choices. For some extreme cases however, namely where $\eta<<1$ and $\gamma>>1$, and where $\eta>>1$ and $\gamma<<1, \theta_{1}$ and $\beta_{1}$ can become positive and negative respectively, and the first solutions in Eqs. (49) and (50) become the correct choices. A more detailed explanation will be given in Section 2.5.1. Also note the reversal of the sign in Eq. (50), which is due to the different directions of $D_{s}$ in Fig. 1b and in Refs. [25-34]. Eqs. (49) and (50) now allow the evaluation of $S_{n}$ and $S_{s}$ in Eqs. (39) and (42).

\subsection{Determining the ERRs, $G_{I}$ and $G_{I I}$}

It is seen either from Eq. (18), or from Eqs. (43) and (44), that $K_{I}$ and $K_{I I}$ can be determined if $G_{I}$ and $G_{I I}$ are known first. A powerful methodology has been developed in the authors' previous work [25-34] for mixed-mode partitions based on orthogonal pure modes. Once one pure mode has been found-by analytical, numerical or experimental means-the other pure modes can be determined analytically by using the orthogonality property between them. The ERRs, $G_{I}$ and $G_{I I}$, for a general loading condition can then be calculated by using these pure modes. Readers are referred to Refs. [25-34] for a detailed description of the methodology, which is also used here.

The methodology starts by considering the bimaterial DCB shown in Fig. 1a but with only the two crack tip bending moments, $M_{1 B}$ and $M_{2 B}$, and no other loads. The aim is to find the pure mode I relationship between these crack tip bending moments, that is, to find $\theta_{1}$ in the relationship $M_{2 B}=\theta_{1} M_{1 B}$. Note that $\theta_{1}$ must be a function of the thickness ratio $\gamma$, the modulus ratio $\eta$ and Poisson's ratio $v$. In many engineering contexts, the Poisson's ratios of the top and bottom layers, $v_{1}$ and $v_{2}$, are close to each other [44], that is, $v_{1} \approx v_{2}$. Therefore in the following, $v_{1}=v_{2}=v$ is assumed. Also it is worth nothing that in many of these cases $v \approx 1 / 3$ [44] although the assumption is not required in this work. Furthermore, since 2D-elastic bimaterial problems depend only on the two Dundurs parameters [44], for a given modulus ratio $\eta$, a single equivalent Poisson's ratio for both layers combined can be found that gives the same values of the Dundurs parameters. It is anticipated that this will allow cases where $v_{1} \neq v_{2}$ to be considered using theory presented in this paper. More details on this will be reported in a future paper by the authors. 
Because of the violent oscillation of interfacial stresses near to the crack tip in Eqs. (1) to (3), the value of $\theta_{1}$ is crack extension size-dependent or FEM mesh size-dependent. In the following, an analytical $\theta_{1}$ is found for a crack extension size of $\delta a=0.05$ at which it is seen from Fig. 3 that $\theta_{1}$ and $\theta_{1}^{\prime}$ approximately coincide. This is achieved by considering the ERR partition $G_{I} / G$ based on the crack extension size $\delta a=0.05$ with the loading condition $M_{2 B} / M_{1 B}=0$ versus the modulus ratio $\eta$ for different thickness ratios $\gamma$ and Poisson's ratios $v$. 2D FEM simulation results are presented for these loading conditions in Fig. 4 for $v=0.29$. Similar graphs can be obtained for different Poisson's ratios. The results from the authors' Timoshenko beam partition theory [25-34] are also presented for the $\gamma=1$ case, denoted by the thick black line which is labelled $\left(\mathrm{G}_{\mathrm{IT}} / G\right)_{\gamma=1, \eta}$. It is interesting to note that the 2D FEM simulation results for this $\gamma=1$ case approximately coincide with the results from the authors' Timoshenko beam partition theory. Furthermore, Fig. 4 shows that when $M_{2 B} / M_{1 B}=0$, the 2D FEM partition results correspond to a non-uniform vertical shift of the Timoshenko beam partition results with $\gamma=1$. These observations lead to the determination of the pure mode I $\theta_{1}(\gamma, \eta, v)$ mode by means of a shifting technique. From Fig. 4, it is seen that when $\gamma<1$ each of the curves is easily distinguishable, whereas when $\gamma>1$ the curves are closely grouped together. It is anticipated that this tight grouping would lead to inaccuracies and high sensitivity in the shifting technique. Therefore the shifting technique is developed for $\gamma \leq 1$ only. Consideration of physical symmetry in Section 2.5.2 allows the method to be used for $\gamma>1$.

\subsubsection{Pure mode I $\theta_{1}(\gamma, \eta, v)$ when $\gamma \leq 1$}

By considering Fig. 4 , the partition $\left(G_{\mathrm{I}} / G\right)_{\gamma \cdot \eta}$ for the loading case $M_{2 B} / M_{1 B}=0$, based on a non-uniform vertical shift $S(\gamma, \eta, v)$, can be written as

$$
\left(\frac{G_{I}}{G}\right)_{\gamma, \eta, v}=\left(\frac{G_{I T}}{G}\right)_{\gamma=1, \eta}+S(\gamma, \eta, v)
$$

where $\left(G_{I T} / G\right)_{\gamma=1, \eta}$ is the partition of ERR from Timoshenko beam theory [25-34] with $\gamma=1$ and variable $\eta$, and is given by

$$
\left(\frac{G_{I T}}{G}\right)_{\gamma=1, \eta}=\frac{(7+\eta)^{2}}{4(1+\eta)(13+\eta)}
$$


Since $\left(G_{\mathrm{I}} / G\right)_{\gamma, \eta, v}$ obtained from Eq. (51) in terms of the shift $S$ must be the same as $\left(G_{\mathrm{I}} / G\right)_{\gamma, \eta, v}$ given by Eq. (47) with $M_{2 B} / M_{1 B}=0$, which is in terms of $\theta_{1}$, then

$$
\left(\frac{G_{I T}}{G}\right)_{\gamma=1, \eta}+S=\frac{C_{I} M_{1 B}^{2}}{G}
$$

where $G=C_{11} M_{1 B}{ }^{2} /\left(2 \bar{E}_{1} b^{2}\right)$, as given by Eq. (A5) with $M_{2 B}=N_{1 B}=N_{2 B}=0$, and $C_{I}$ is given by Eq. (A1) with $\beta_{1}^{\prime}=\beta_{1}$ (since $\delta a=0.05$ ). Note that $\beta_{1}$ is orthogonal to $\theta_{1}$ (see Section 2.5.3) and that therefore $\beta_{1}=-\left(C_{11}+C_{12} \theta_{1}\right) /\left(C_{12}+C_{22} \theta_{1}\right)$ with $C_{i j}$ given in Appendix B. The resulting relationship between $\theta_{1}$ and $S$ is then obtained as

$$
\frac{C_{11}}{2 \bar{E}_{1} b^{2}}\left[\left(\frac{G_{I T}}{G}\right)_{\gamma=1, \eta}+S\right]=G_{\theta_{1}}\left[\frac{C_{11}+C_{12} \theta_{1}}{C_{22} \theta_{1}^{2}+2 C_{12} \theta_{1}+C_{11}}\right]^{2}
$$

where $G_{\theta_{1}}$ is given by Eq. (A3). Eq. (54) can now be solved for $\theta_{1}$ in terms of the shift $S$, which gives two possible solutions. Using the orthogonality condition between $\theta_{1}$ and $\beta_{1}$, there are also two corresponding solutions for $\beta_{1}$. The algebraic expression for each solution is too long to be presented here, however Eq. (54) is easy to solve for numerical values of $\gamma, \eta$ and $S$. To proceed, it is essential that the correct solutions for $\theta_{1}$ and $\beta_{1}$ are determined. To do this, consider Fig. 4. It is seen that for some values of $\gamma$, there exists a value of $\eta$, denoted here by $\eta_{\beta}$, at which the partition $G_{\mathrm{I}} / G$ reaches 0 , that is, when $\eta=\eta_{\beta}$ then $\beta_{1}=0$. Therefore for $\eta<\eta_{\beta}$, or $\log _{10}(1 / \eta)>\log _{10}\left(1 / \eta_{\beta}\right)$, choose the positive solution for $\beta_{1}$ from Eq. (54). For $\eta>\eta_{\beta}$, or $\log _{10}(1 / \eta)<\log _{10}\left(1 / \eta_{\beta}\right)$, choose the negative solution. If $\eta_{\beta}$ does not exist, then the positive solution must also be chosen. When the shift $S$ has been determined, as it will be shortly, then Eq. (51) can easily be solved with $\left(G_{\mathrm{I}} / G\right)_{\gamma, \eta, \nu}=0$ to determine the numerical value of $\eta_{\beta}$.

The above procedure for choosing the correct solution for $\beta_{1}$ works very well when the two solutions have opposite signs, which is usually the case. However, when $\gamma$ becomes close to 1 , sometimes the two solutions for $\beta_{1}$ have the same sign. When this happens, it is still easy to determine the correct solution because when $\gamma \approx 1$ then $\theta_{1} \approx-\eta \gamma^{2}$ and $\beta_{1} \approx \eta \gamma^{2}\left(\eta \gamma^{2}+4 \gamma+3\right) /\left(3 \eta \gamma^{2}+4 \eta \gamma+1\right)$, which are the values based on Timoshenko beam theory. The pair of solutions which are closest to these values are the correct ones. 
Now more detailed explanations for Eqs. (49) and (50) can be given. Consider Eq. (49) with $M_{1 B}>0$ and $M_{2 B}=N_{1 B}=N_{2 B}=0$. Since $D_{n}$ is positive when $\eta<\eta_{\beta}$, or $\log _{10}(1 / \eta)>\log _{10}\left(1 / \eta_{\beta}\right)$, and $D_{n}=0$ when $\eta=\eta_{\beta}$, therefore when $\eta>\eta_{\beta}$, or $\log _{10}(1 / \eta)<\log _{10}\left(1 / \eta_{\beta}\right)$, then $D_{n}$ must be negative. A similar argument can be used for Eq. (50).

It now remains to find the non-uniform vertical shift $S$ for $\gamma \leq 1$. The shift $S$ is assumed to be in the following form:

$$
S(\gamma, \eta, v)=S_{0}(\gamma, v)+S_{1}(\gamma, v) \log _{10}\left(\frac{1}{\eta}\right)+S_{2}(\gamma, v)\left[\log _{10}\left(\frac{1}{\eta}\right)\right]^{2}
$$

The coefficients, $S_{0}, S_{1}$ and $S_{2}$, are determined empirically by considering $\eta=1,1 / 10,1 / 100,10,100$ in Eqs. (51) and (55). Doing so gives

$$
\begin{gathered}
S_{0}(v)=\left(\frac{G_{I}}{G}\right)_{\gamma, \eta=1}-\left(\frac{G_{I T}}{G}\right)_{\gamma=1, \eta=1} \\
S_{1}(v)+S_{2}(v)=\left(\frac{G_{I}}{G}\right)_{\gamma, \eta=1 / 10, v}-\left(\frac{G_{I T}}{G}\right)_{\gamma=1, \eta=1 / 10}-S_{0} \\
S_{1}(v)+2 S_{2}(v)=\frac{1}{2}\left[\left(\frac{G_{I}}{G}\right)_{\gamma, \eta=1 / 100, v}-\left(\frac{G_{I T}}{G}\right)_{\gamma=1, \eta=1 / 100}-S_{0}\right] \\
S_{2}(v)-S_{1}(v)=\left(\frac{G_{I}}{G}\right)_{\gamma, \eta=10, v}-\left(\frac{G_{I T}}{G}\right)_{\gamma=1, \eta=10}-S_{0} \\
2 S_{2}(v)-S_{1}(v)=\frac{1}{2}\left[\left(\frac{G_{I}}{G}\right)_{\gamma, \eta=100, v}-\left(\frac{G_{I T}}{G}\right)_{\gamma=1, \eta=100}-S_{0}\right]
\end{gathered}
$$

Ref. [33] provides an accurate expression for $\left(G_{I} / G\right)_{\gamma, \eta=1}$ in Eq. (56), which is not dependent on $v$. It is summarized in Appendix C. 2D FEM simulations with the loading condition $M_{2 B} / M_{1 B}=0$ provide values for $\left(G_{I} / G\right)_{\gamma, \eta=1 / 10, v},\left(G_{I} / G\right)_{\gamma, \eta=1 / 100, v},\left(G_{I} / G\right)_{\gamma, \eta=10, v}$ and $\left(G_{I} / G\right)_{\gamma, \eta=100, v}$ for different values of $\gamma$ and $v$. When used in conjunction with Eqs. (56) to (60), the following accurate empirical formulae are obtained for $S_{1}$ and $S_{2}$ with $\gamma \leq 1$ :

$$
\begin{aligned}
& S_{1}=S_{13}(v)\left[\log _{10}(1 / \gamma)\right]^{3}+S_{12}(v)\left[\log _{10}(1 / \gamma)\right]^{2}+S_{11}(v) \log _{10}(1 / \gamma)+S_{10} \\
& S_{2}=S_{23}(v)\left[\log _{10}(1 / \gamma)\right]^{3}+S_{22}(v)\left[\log _{10}(1 / \gamma)\right]^{2}+S_{21}(v) \log _{10}(1 / \gamma)+S_{20}
\end{aligned}
$$


where $S_{i j}$ in the plane strain condition are given by

$$
\begin{aligned}
& S_{13}= \begin{cases}-0.179784 v^{2}+0.150620 v-0.045250 & \text { if } \eta<1 \\
-1.707125 v^{2}+0.098035 v+0.327941 & \text { if } \eta>1\end{cases} \\
& S_{12}= \begin{cases}0.487995 v^{2}-0.208470 v+0.024941 & \text { if } \eta<1 \\
3.145748 v^{2}-0.592065 v-0.653980 & \text { if } \eta>1\end{cases} \\
& S_{11}= \begin{cases}-0.404560 v^{2}-0.109477 v+0.084873 & \text { if } \eta<1 \\
-1.510591 v^{2}+0.668378 v+0.394354 & \text { if } \eta>1\end{cases} \\
& S_{10}= \begin{cases}-1.339729 v^{2}-0.112935 v+0.164729 & \text { if } \eta<1 \\
-1.387396 v^{2}-0.281472 v+0.219649 & \text { if } \eta>1\end{cases} \\
& S_{23}=\left\{\begin{array}{cc}
0.055138 v^{2}-0.054976 v+0.010606 & \text { if } \eta<1 \\
-1.184839 v^{2}+0.480577 v+0.139204 & \text { if } \eta>1
\end{array}\right. \\
& S_{22}=\left\{\begin{array}{cc}
-0.154013 v^{2}+0.107145 v-0.004940 & \text { if } \eta<1 \\
1.591445 v^{2}-0.995923 v-0.201936 & \text { if } \eta>1
\end{array}\right. \\
& S_{21}=\left\{\begin{array}{cc}
0.134731 v^{2}-0.038117 v-0.016633 & \text { if } \eta<1 \\
-0.307568 v^{2}+0.335473 v+0.139806 & \text { if } \eta>1
\end{array}\right. \\
& S_{20}=\left\{\begin{array}{cc}
0.359714 v^{2}+0.087096 v-0.063181 & \text { if } \eta<1 \\
-0.381264 v^{2}-0.090766 v+0.066329 & \text { if } \eta>1
\end{array}\right.
\end{aligned}
$$

and $S_{i j}$ in the plane stress condition are given in Appendix D.

When the crack extension size $\delta a=0.05$ then, as has been seen in Fig. 3, $\theta_{1}^{\prime} \approx \theta_{1}$ and $\beta_{1}^{\prime} \approx \beta_{1}$. Therefore negative $G_{I}$ or $G_{I I}$ does not occur (similarly, neither does $G_{I}>G$ or $G_{I I}>G$ ). The shift $S$ given by Eq. (55) must therefore not result in $G_{I} / G<0$ or $G_{I} / G>1$. Due to small numerical inaccuracies, the shift must be capped in some cases to prevent this from happening. From Eq. (51),

$$
S= \begin{cases}1-\left(\frac{G_{I T}}{G}\right)_{\gamma=1, \eta} & \text { if } S>1-\left(\frac{G_{I T}}{G}\right)_{\gamma=1, \eta} \\ -\left(\frac{G_{I T}}{G}\right)_{\gamma=1, \eta} & \text { if } S<-\left(\frac{G_{I T}}{G}\right)_{\gamma=1, \eta}\end{cases}
$$




\subsubsection{Pure mode I $\theta_{1}(\gamma, \eta, v)$ when $\gamma>1$}

Because of physical symmetry, it is a requirement that

$$
\theta_{1}(\gamma, \eta, v)=\theta_{1}^{-1}\left(\gamma^{-1}, \eta^{-1}, v\right)
$$

Therefore, when $\gamma>1, \theta_{1}(\gamma, \eta, v)$ can be found by first finding $\theta_{1}\left(\gamma^{-1}, \eta^{-1}, v\right)$ with the method in Section 2.5.1, and then using Eq. (72).

\subsubsection{The complete set of pure mode I $\theta_{i}$ modes and pure mode II $\beta_{i}$ modes}

The pure mode I $\theta_{i}$ modes and the pure mode II $\beta_{i}$ modes (with $i=1,2,3$ ), which are required in Eqs. (47) and (48), are determined using the orthogonal methodology [25-34]. The pure mode II $\beta_{1}$ mode is orthogonal to the pure mode I $\theta_{1}$ mode. This is written as

$$
\beta_{1}=\operatorname{orthogonal}\left(\theta_{1}\right)
$$

which is equivalent to

$$
0=\left\{\begin{array}{llll}
1 & \theta_{1} & 0 & 0
\end{array}\right\}[C]\left\{\left\{\begin{array}{llll}
1 & \beta_{1} & 0 & 0
\end{array}\right\}^{T}\right.
$$

where $[C]$ is given by Eqs. (A5) to (A16). Therefore if $\theta_{1}$ is known, then $\beta_{1}$ can easily be determined. Similarly,

$$
\begin{aligned}
& \theta_{2}=\operatorname{orthogonal}\left(\beta_{1}\right) \quad \text { or } \quad 0=\left\{\begin{array}{llll}
1 & 0 & \theta_{2} & 0
\end{array}\right\}[C]\left\{\begin{array}{llll}
1 & \beta_{1} & 0 & 0
\end{array}\right\}^{T} \\
& \theta_{3}=\operatorname{orthogonal}\left(\beta_{1}\right) \quad \text { or } \quad 0=\left\{\begin{array}{llll}
1 & 0 & 0 & \theta_{3}
\end{array}\right\}[C]\left\{\left\{\begin{array}{llll}
1 & \beta_{1} & 0 & 0
\end{array}\right\}^{T}\right. \\
& \beta_{2}=\text { orthogonal }\left(\theta_{1}\right) \quad \text { or } \quad 0=\left\{\begin{array}{llll}
1 & 0 & \beta_{2} & 0
\end{array}\right\}[C]\left\{\begin{array}{llll}
1 & \theta_{1} & 0 & 0
\end{array}\right\}^{T} \\
& \beta_{3}=\operatorname{orthogonal}\left(\theta_{1}\right) \quad \text { or } \quad 0=\left\{\begin{array}{llll}
1 & 0 & 0 & \beta_{3}
\end{array}\right\}[C]\left\{\begin{array}{llll}
1 & \theta_{1} & 0 & 0
\end{array}\right\}^{T}
\end{aligned}
$$

The ERRs, $G_{I}$ and $G_{I I}$, at a crack extension size of $\delta a=0.05$ can now be obtained from Eqs. (47) and (48). The various pairs of mathematically admissible SIFs, $K_{I}$ and $K_{I I}$ can be obtained from Eqs. (18) to (24). The only mechanically admissible pair is chosen by using Eqs. (43) and (44) as a guide. The ERR partitions, $G_{I}$ and $G_{I I}$, for all crack extension sizes, $\delta a$, can then be calculated from Eqs. (13) and (14) or from Eqs. (25) and (26).

\section{Conclusions}

The discoveries and conclusions are now summarized below: 
(1) Material mismatch causes the existence of two distinct sets of orthogonal pure modes, $\left(\theta_{K}, \beta_{K}\right)$ and $\left(\theta_{K}^{\prime}, \beta_{K}^{\prime}\right)$, and two sets of coincident orthogonal approximate pure modes, $\left(\tilde{\theta}_{K}, \tilde{\beta}_{K}\right)$ and $\left(\tilde{\theta}_{K}^{\prime}, \tilde{\beta}_{K}^{\prime}\right)$, which are in terms of the SIFs, $K_{I}$ and $K_{I I}$, and which are crack extension sizedependent or FEM mesh size-dependent. The total ERR $G$ can be partitioned by using these pure modes. In general, for cracks on a bimaterial interface, there are four pairs of mathematically admissible SIFs, $K_{I}$ and $K_{I I}$, for a given loading condition. Only one pair, however, is mechanically admissible and it has been analytically determined.

(2) A brittle interface causes the existence of two distinct sets of orthogonal pure modes, $\left(\theta_{i}, \beta_{i}\right)$ and $\left(\theta_{i}^{\prime}, \beta_{i}^{\prime}\right)$ (with $\left.i=1,2,3\right)$, which are in terms of the crack tip loads. In the case of interfacial cracks between similar materials, these two sets of pure modes approach to each other and remain converged with the diminishing global effect as the crack extension size or FEM mesh size decreases. In the case of cracks on bimaterial interfaces, although these two sets of pure modes also approach to each other and become coincident as the crack extension size or FEM mesh size decreases, they do not converge and are crack extension size-dependent or FEM mesh size-dependent. Furthermore, they separate again for very small crack extension sizes or FEM mesh sizes.

(3) At a crack extension size $\delta a=0.05$, a thickness ratio $\gamma=1$ and Poisson's ratio $v=0.29$, the distinct two sets of orthogonal pure modes, $\left(\theta_{i}, \beta_{i}\right)$ and $\left(\theta_{i}^{\prime}, \beta_{i}^{\prime}\right)$ (with $i=1,2,3$ ), approximately coincide with each other and are also approximately equal to the pure modes based on Timoshenko beam theory for entire range of modulus ratio $1 / 100 \leq \eta \leq 100$. A shifting technique has been developed and used in conjunction with the authors' orthogonal pure mode methodology to determine the pure modes for Poisson's ratio in the range $0 \leq v \leq 0.5$, Young's modulus ratio in the range $1 / 100 \leq \eta \leq 100$, and thickness ratio in the range $1 / 10 \leq \gamma \leq 10$. Consequently, the SIFs, $K_{I}$ and $K_{I I}$, and the crack extension size-dependent ERRs, $G_{I}$ and $G_{I I}$, are analytically determined.

The analytical theory will be verified in Part 2 [35] and further conclusions will be made there.

\section{Appendix A}

In Eqs. (45) and (46), 


$$
\begin{aligned}
& c_{I}=G_{\theta_{1}}\left[\left(1-\frac{\theta_{1}}{\beta_{1}}\right)\left(1-\frac{\theta_{1}}{\beta_{1}^{\prime}}\right)\right]^{-1} \\
& c_{I I}=G_{\beta_{1}}\left[\left(1-\frac{\beta_{1}}{\theta_{1}}\right)\left(1-\frac{\beta_{1}}{\theta_{1}^{\prime}}\right)\right]^{-1}
\end{aligned}
$$

where

$$
\begin{aligned}
G_{\theta_{1}} & =6\left[\theta_{1}^{2}\left(3 \eta \gamma^{3}+6 \eta \gamma^{2}+4 \eta \gamma+1\right)-\theta_{1}\left(2 \eta^{2} \gamma^{4}+2 \eta \gamma^{3}\right)\right. \\
& \left.+\left(\eta^{3} \gamma^{7}+4 \eta^{2} \gamma^{6}+6 \eta^{2} \gamma^{5}+3 \eta^{2} \gamma^{4}\right)\right] /\left[\bar{E}_{1} b^{2} \eta \gamma^{3} h_{1}^{3}\left(\eta^{2} \gamma^{4}+4 \eta \gamma^{3}+6 \eta \gamma^{2}+4 \eta \gamma+1\right)\right] \\
G_{\beta_{1}} & =6\left[\beta_{1}^{2}\left(3 \eta \gamma^{3}+6 \eta \gamma^{2}+4 \eta \gamma+1\right)-\beta_{1}\left(2 \eta^{2} \gamma^{4}+2 \eta \gamma^{3}\right)\right. \\
& \left.+\left(\eta^{3} \gamma^{7}+4 \eta^{2} \gamma^{6}+6 \eta^{2} \gamma^{5}+3 \eta^{2} \gamma^{4}\right)\right] /\left[\bar{E}_{1} b^{2} \eta \gamma^{3} h_{1}^{3}\left(\eta^{2} \gamma^{4}+4 \eta \gamma^{3}+6 \eta \gamma^{2}+4 \eta \gamma+1\right)\right]
\end{aligned}
$$

and $\bar{E}_{1}$ is the effective Young's modulus of the top beam. For plane stress then $\bar{E}_{1}=E_{1}$ and for plane strain then $\bar{E}_{1}=E_{1} /\left(1-v_{1}^{2}\right)$.

\section{Appendix B}

With reference to Fig. 1a, the total ERR $G$ of a bimaterial DCB with two crack tip bending moments, $M_{1 B}$ and $M_{2 B}$, and two crack tip axial forces, $N_{1 B}$ and $N_{2 B}$, is given by

$$
G=\frac{1}{2 \bar{E}_{1} b^{2}}\left\{\begin{array}{c}
M_{1 B} \\
M_{2 B} \\
N_{1 B} \\
N_{2 B}
\end{array}\right\}^{T}\left[\begin{array}{llll}
C_{11} & C_{12} & C_{13} & C_{14} \\
C_{12} & C_{22} & C_{23} & C_{24} \\
C_{13} & C_{23} & C_{33} & C_{34} \\
C_{14} & C_{24} & C_{34} & C_{44}
\end{array}\right]\left\{\begin{array}{c}
M_{1 B} \\
M_{2 B} \\
N_{1 B} \\
N_{2 B}
\end{array}\right\}=\frac{1}{2 \bar{E}_{1} b^{2}}\left\{\begin{array}{c}
M_{1 B} \\
M_{2 B} \\
N_{1 B} \\
N_{2 B}
\end{array}\right\}[C]\left\{\begin{array}{c}
M_{1 B} \\
M_{2 B} \\
N_{1 B} \\
N_{2 B}
\end{array}\right\}
$$

where $[C]$ is the coefficient matrix, given by

$$
\begin{gathered}
C_{11}=\frac{12 \eta \gamma\left(\eta \gamma^{3}+4 \gamma^{2}+6 \gamma+3\right)}{h_{1}^{3} \bar{C}} \\
C_{12}=-\frac{12(\eta \gamma+1)}{h_{1}^{3} \bar{C}} \\
C_{13}=\frac{6 \eta \gamma(\gamma+1)}{h_{1}{ }^{2} \bar{C}} \\
C_{14}=-\frac{6(\gamma+1)}{h_{1}{ }^{2} \bar{C}}
\end{gathered}
$$




$$
\begin{gathered}
C_{22}=\frac{12\left(3 \eta \gamma^{3}+6 \eta \gamma^{2}+4 \eta \gamma+1\right)}{\eta h_{1}^{3} \gamma^{3} \bar{C}} \\
C_{23}=\frac{6 \eta \gamma(\gamma+1)}{h_{1}^{2} \bar{C}} \\
C_{24}=-\frac{6(\gamma+1)}{h_{1}^{2} \bar{C}} \\
C_{33}=\frac{\eta \gamma\left(\eta \gamma^{3}+1\right)}{h_{1} \bar{C}} \\
C_{34}=-\frac{\eta \gamma^{3}+1}{h_{1} \bar{C}} \\
C_{44}=\frac{\eta \gamma^{3}+1}{\eta h_{1} \bar{C}^{2}} \\
\bar{C}=\eta^{2} \gamma^{4}+4 \eta \gamma^{3}+6 \eta \gamma^{2}+4 \eta \gamma+1
\end{gathered}
$$

\section{Appendix C}

Ref. [33] provides an accurate method to calculate the 2D elasticity-based ERR partitions, $G_{I}$ and $G_{I I}$, when $\eta=1$. Ref. [33] gives $\theta_{1}$ as

$$
\theta_{1}=-\gamma^{2}\left[\frac{(1+\gamma)^{2}+c_{\theta}}{(1+\gamma)^{2}+c_{\theta} \gamma^{2}}\right]
$$

where

$$
c_{\theta}=\bar{c}_{\theta}\left[2-\hat{c}_{\beta}^{0.5}\right]
$$

and $\bar{c}_{\theta}=6 / 5$ and $\hat{c}_{\beta}=(1+\gamma)^{3} /\left(1+\gamma^{3}\right)$. The pure mode I $\theta_{i}$ modes and the pure mode II $\beta_{i}$ modes (with $i=1,2$ ) are determined by using the orthogonal methodology [25-34] (see Section 2.5.3). Since $\eta=1$, the coefficient matrix $[C]$ of the total ERR $G$, is now given by

$$
[C]=\frac{1}{\bar{E} b^{2} h_{1}^{3} \gamma^{3}(1+\gamma)^{3}}\left[\begin{array}{ccc}
6 \gamma^{4}\left(\gamma^{2}+3 \gamma+3\right) & -6 \gamma^{3} & 3 h_{1} \gamma^{4} \\
-6 \gamma^{3} & 6\left(3 \gamma^{2}+3 \gamma+1\right) & 3 h_{1} \gamma^{4} \\
3 h_{1} \gamma^{4} & 3 h_{1} \gamma^{4} & h_{1}^{2} \gamma^{4}\left(\gamma^{2}-\gamma+1\right) / 2
\end{array}\right]
$$

The ERR partitions, $G_{I}$ and $G_{I I}$, are then obtained from 


$$
\begin{aligned}
G_{I} & =c_{I}\left(M_{1 B}-\frac{M_{2 B}}{\beta_{1}}-\frac{N_{1 B e}}{\beta_{2}}\right)\left(M_{1 B}-\frac{M_{2 B}}{\beta_{1}^{\prime}}-\frac{N_{1 B e}}{\beta_{2}^{\prime}}\right) \\
G_{I I} & =c_{I I}\left(M_{1 B}-\frac{M_{2 B}}{\theta_{1}}-\frac{N_{1 B e}}{\theta_{2}}\right)\left(M_{1 B}-\frac{M_{2 B}}{\theta_{1}^{\prime}}-\frac{N_{1 B e}}{\theta_{2}^{\prime}}\right)
\end{aligned}
$$

where $N_{1 B e}=N_{1 B}-N_{2 B} / \gamma$.

\section{Appendix D}

The empirical coefficients $S_{i j}$ in the plane stress condition in Eqs. (61) and (62) are given by

$$
\begin{aligned}
& S_{13}= \begin{cases}-0.047964 v^{2}+0.091462 v-0.045410 & \text { if } \eta<1 \\
-0.316860 v^{2}-0.142473 v+0.333225 & \text { if } \eta>1\end{cases} \\
& S_{12}= \begin{cases}0.086063 v^{2}-0.084216 v+0.024542 & \text { if } \eta<1 \\
0.701381 v^{2}-0.074332 v-0.666427 & \text { if } \eta>1\end{cases} \\
& S_{11}=\left\{\begin{array}{cc}
0.021738 v^{2}-0.170242 v+0.085179 & \text { if } \eta<1 \\
-0.465320 v^{2}+0.358274 v+0.402455 & \text { if } \eta>1
\end{array}\right. \\
& S_{10}= \begin{cases}-0.088802 v^{2}-0.327669 v+0.170305 & \text { if } \eta<1 \\
-0.018717 v^{2}-0.486112 v+0.224870 & \text { if } \eta>1\end{cases} \\
& S_{23}=\left\{\begin{array}{cc}
0.007841 v^{2}-0.032222 v+0.010789 & \text { if } \eta<1 \\
-0.388178 v^{2}+0.264900 v+0.144129 & \text { if } \eta>1
\end{array}\right. \\
& S_{22}=\left\{\begin{array}{cc}
-0.028062 v^{2}+0.057052 v-0.004924 & \text { if } \eta<1 \\
0.627350 v^{2}-0.649216 v-0.210480 & \text { if } \eta>1
\end{array}\right. \\
& S_{21}=\left\{\begin{array}{cc}
0.018448 v^{2}-0.006505 v-0.016887 & \text { if } \eta<1 \\
-0.153962 v^{2}+0.244120 v+0.142364 & \text { if } \eta>1
\end{array}\right. \\
& S_{20}=\left\{\begin{array}{cc}
-0.004950 v^{2}+0.139969 v-0.064582 & \text { if } \eta<1 \\
0.005332 v^{2}-0.147687 v+0.067805 & \text { if } \eta>1
\end{array}\right.
\end{aligned}
$$

\section{References}

[1] Williams ML. The stresses around a fault or crack in dissimilar media. Bulletin of the Seismological Society of America 1959;49:199-204. 
[2] Erdogan F. Stress distribution in a nonhomogeneous elastic plane with cracks. Journal of Applied Mechanics 1963;30:232-236.

[3] Erdogan F. Stress distribution in bonded dissimilar materials with cracks. Journal of Applied Mechanics 1965;32:403-410.

[4] Rice JR, Sih GC. Plane problems of cracks in dissimilar media. Journal of Applied Mechanics 1965;32:418-423.

[5] England AH. A crack between dissimilar media. Journal of Applied Mechanics 1965;32:400-402.

[6] Comninou M. The interface crack. Journal of Applied Mechanics 1977;44:631-636.

[7] Comninou M. The interface crack in a shear field. Journal of Applied Mechanics 1978;45:287-290.

[8] Comninou M, Schmueser D. The interface crack in a combined tension-compression and shear field. Journal of Applied Mechanics 1979;46:345-348.

[9] Gautesen AK, Dundurs J. The interface crack in a tension field. Journal of Applied Mechanics 1987;54:93-98.

[10] Gautesen AK, Dundurs J. The interface crack under combined loading. Journal of Applied Mechanics 1988;55:580-586.

[11] Gautesen AK. The interface crack under combined loading: an eigenvalue problem for the gap. International Journal of Fracture 1993;60:349-361.

[12] Sun CT, Qian W. The use of finite extension strain energy release rates in fracture of interfacial cracks. International Journal of Solids and Structures 1997;34:2595-2609.

[13] Rice JR. Elastic fracture mechanics concepts for interfacial cracks. Journal of Applied Mechanics 1988;55:98-103.

[14] Hutchinson JW, Mear ME, Rice JR. Crack paralleling an interface between dissimilar materials. Journal of Applied Mechanics 1987;54:828-832.

[15] Sun CT, Jih CJ. On strain energy release rates for interfacial cracks in bi-material media. Engineering Fracture Mechanics 1987;28:13-20.

[16] Raju IS, Crews Jr JH, Aminpour MA. Convergence of strain energy release rate components for edge-delaminated composite laminates. Engineering Fracture Mechanics 1988;30:383-396. 
[17] Chow WT, Atluri SN. Finite element calculation of stress intensity factors for interfacial crack using virtual crack closure integral. Computational Mechanics 1995;16:417-425.

[18] Parmigiani JP, Thouless MD. The effects of cohesive strength and toughness on mixedmode delamination of beam-like geometries. Engineering Fracture Mechanics 2007;74:2675-2699.

[19] Morioka Y, Sun CT. A simple method for determining stress intensity factors in bi-material interfacial cracks. In: Proceedings of the 51st AIAA/ASME/ASCE/AHS/ASC Structures, Structural Dynamics, and Materials Conference, 12-15 April 2010, Orlando, Florida, USA.

[20] Suo Z, Hutchinson JW. Interface crack between two elastic layers. International Journal of Fracture 1990;43:1-18.

[21] Zhou YC, Tonomori T, Yoshida A, Liu L, Bignall G, Hashida T. Fracture characteristics of thermal barrier coatings after tensile and bending tests. Surface and Coatings Technology 2002;157:118-127.

[22] Deng HX, Shi HJ, Yu HC, Zhong B. Determination of mixed-mode interfacial fracture toughness for thermal barrier coatings. Science China Physics, Mechanics and Astronomy 2011;54:618-624.

[23] Fleck NA, Cocks ACF, Lampenscherf S. Thermal shock resistance of air plasma sprayed thermal barrier coatings. Journal of the European Ceramic Society 2014;34:2687-2694.

[24] Arai M, Okajima Y, Kishimoto K. Mixed-mode interfacial fracture toughness for thermal barrier coating. Engineering Fracture Mechanics 2007;74:2055-2069.

[25] Wang S, Harvey CM. Mixed mode partition in one dimensional fracture. Journal of Key Engineering Materials 2011;462-63:616-621. Also a plenary lecture in the 8th International Conference on Fracture and Strength of Solids (FEOFS 2010), 7-9th June 2010, Kuala Lumpur, Malaysia.

[26] Harvey CM. Mixed-mode partition theories for one-dimensional fracture. PhD Thesis. March 2012, Loughborough University, UK.

[27] Wang S, Guan L. On fracture mode partition theories. Computational Material Sciences 2012;52:240-245.

[28] Wang S, Harvey CM. A theory of one-dimensional fracture. Composite Structures 2012;94:758-767. Also a plenary lecture at the 16th International Conference on Composite Structures (ICCS16), 28-30 June 2011, Porto, Portugal. 
[29] Harvey CM, Wang S. Experimental assessment of mixed-mode partition theories. Composite Structures 2012;94:2057-2067.

[30] Wang S, Harvey CM. Mixed mode partition theories for one dimensional fracture. Engineering Fracture Mechanics 2012;79:329-352.

[31] Harvey CM, Wang S. Mixed-mode partition theories for one-dimensional delamination in laminated composite beams. Engineering Fracture Mechanics 2012;96:737-759.

[32] Wang S, Harvey CM, Guan L. Partition of mixed modes in layered isotropic double cantilever beams with non-rigid cohesive interfaces. Engineering Fracture Mechanics 2013;111:1-25.

[33] Harvey CM, Wood JD, Wang S, Watson A. A novel method for the partition of mixedmode fractures in 2D elastic laminated unidirectional composite beams. Composite Structures 2014;116:589-594.

[34] Harvey CM, Eplett MR, Wang S. Experimental assessment of mixed-mode partition theories for generally laminated composite beams. Composite Structures 2015;124:10-18.

[35] Harvey CM, Wood JD, Wang S. Brittle interfacial cracking between two dissimilar elastic layers: Part 2-Numerical verification. Composite Structures 2015, http://dx.doi.org/10.1016/j.compstruct.2015.06.079

[36] Davidson BD, Fariello PL, Hudson RC, Sundararaman V. Accuracy assessment of the singular-field-based mode-mix decomposition procedure for the prediction of delamination. In: Hooper SJ, editor. Composite materials: testing and design (thirteenth volume), ASTM STP 1242. American Society for Testing and Materials, 1997, pp. 109_ 128.

[37] Davidson BD, Gharibian SJ, Yu LJ. Evaluation of energy release rate-based approaches for predicting delamination growth in laminated composites. International Journal of Fracture 2000;105:343-365.

[38] Davidson BD, Bialaszewski RD, Sainath SS. A non-classical, energy release rate based approach for predicting delamination growth in graphite reinforced laminated polymeric composites. Composites Science and Technology 2006;66:1479-1496.

[39] Hashemi S, Kinloch AJ, Williams JG. The analysis of interlaminar fracture in uniaxial fibre-polymer composites. Proceeding of the Royal Society A 1990;427:173-199.

[40] Kinloch AJ, Wang Y, Williams JG, Yayla P. The mixed-mode delamination of fibre composite materials. Composites Science and Technology 1993;47:225-237. 
[41] Charalambides M, Kinloch AJ, Wang Y, Williams JG. On the analysis of mixed-mode failure. International Journal of Fracture 1992;54:269-291.

[42] Hashemi S, Kinloch AJ, Williams G. Mixed-mode fracture in fiber-polymer composite laminates. In: O’Brien TK, editor. Composite materials: fatigue and fracture (third volume), ASTM STP 1110. Philadelphia, PA: American Society for Testing and Materials, 1991. pp. 143-168.

[43] Conroy M, Sørensen BF, Ivankovic A. Combined numerical and experimental investigation of mode-mixity in beam like geometries. In: Proceedings of the 37th Annual Meeting of the Adhesion Society, Feburary 2014, San Diego, California, USA.

[44] Hutchinson JW, Suo Z. Mixed mode cracking in layered materials. Advances in Applied Mechanics 1991;29:63-191.

[45] Wang S, Harvey CM, Wang B, Watson A. Post-local buckling-driven delamination in bilayer composite beams. Composite Structures 2015 (submitted soon).

[46] Cornetti P, Pugno N, Carpinteri A, Taylor D. Finite fracture mechanics: a coupled stress and energy failure criterion. Engineering Fracture Mechanics 2006;73:2021-2033.

[47] Harvey CM, Wang S. Numerical and analytical study of delamination in composite laminates. International Journal of Engineering Systems Modelling and Simulation 2012;4:120-137.

[48] Zhang Y, Wang S. Buckling, post-buckling and delamination propagation in debonded composite laminates: Part 1 Theoretical development. Composite Structures 2009;88:121130.

[49] Wang S, Zhang Y. Buckling, post-buckling and delamination propagation in debonded composite laminates: Part 2 Numerical applications. Composite Structures 2009;88:131146. 


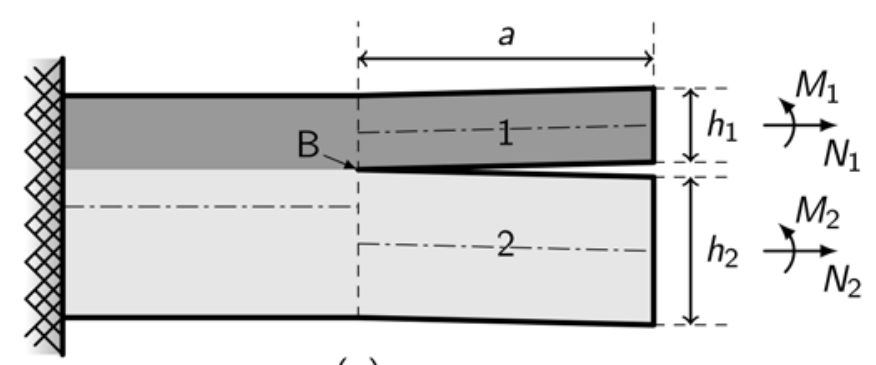

(a)

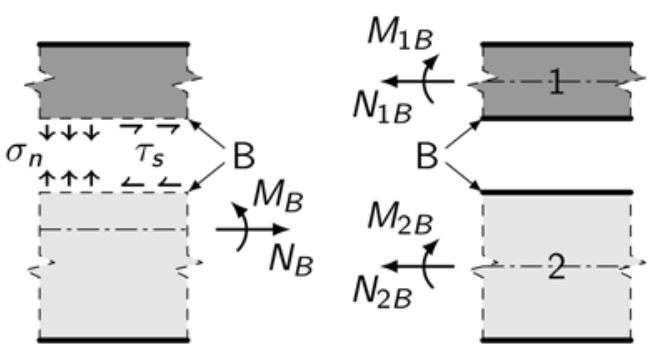

(b)

Fig. 1: A bimaterial DCB. (a) General description. (b) Interfacial stresses and crack tip forces. 


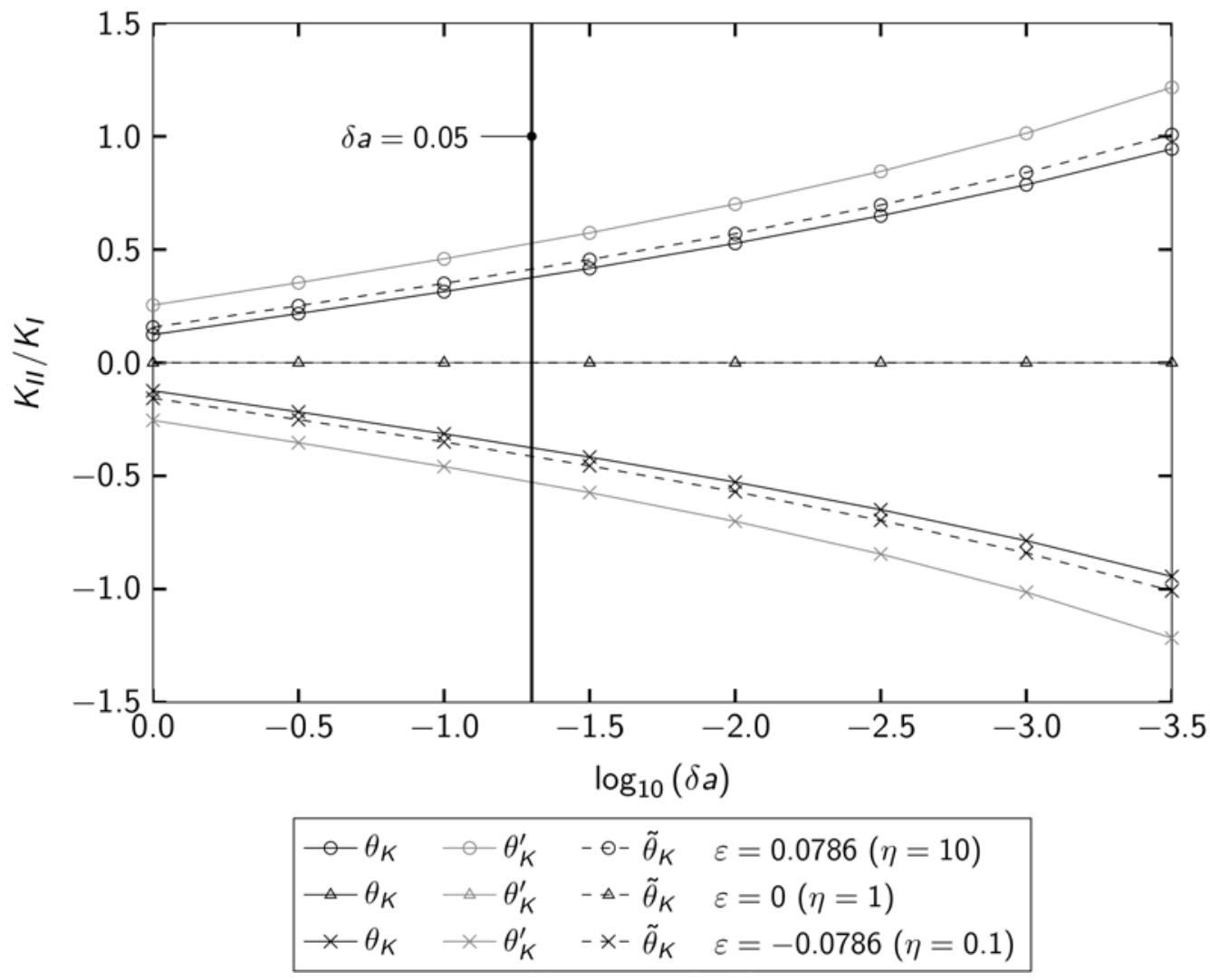

Fig. 2: Variation of $\theta_{K}, \theta_{K}^{\prime}$, and $\tilde{\theta}_{K}$ with respect to the crack extension size $\delta a$ for different values of the bimaterial mismatch constant $\varepsilon$ with $v_{1}=v_{2}=0.29$. 


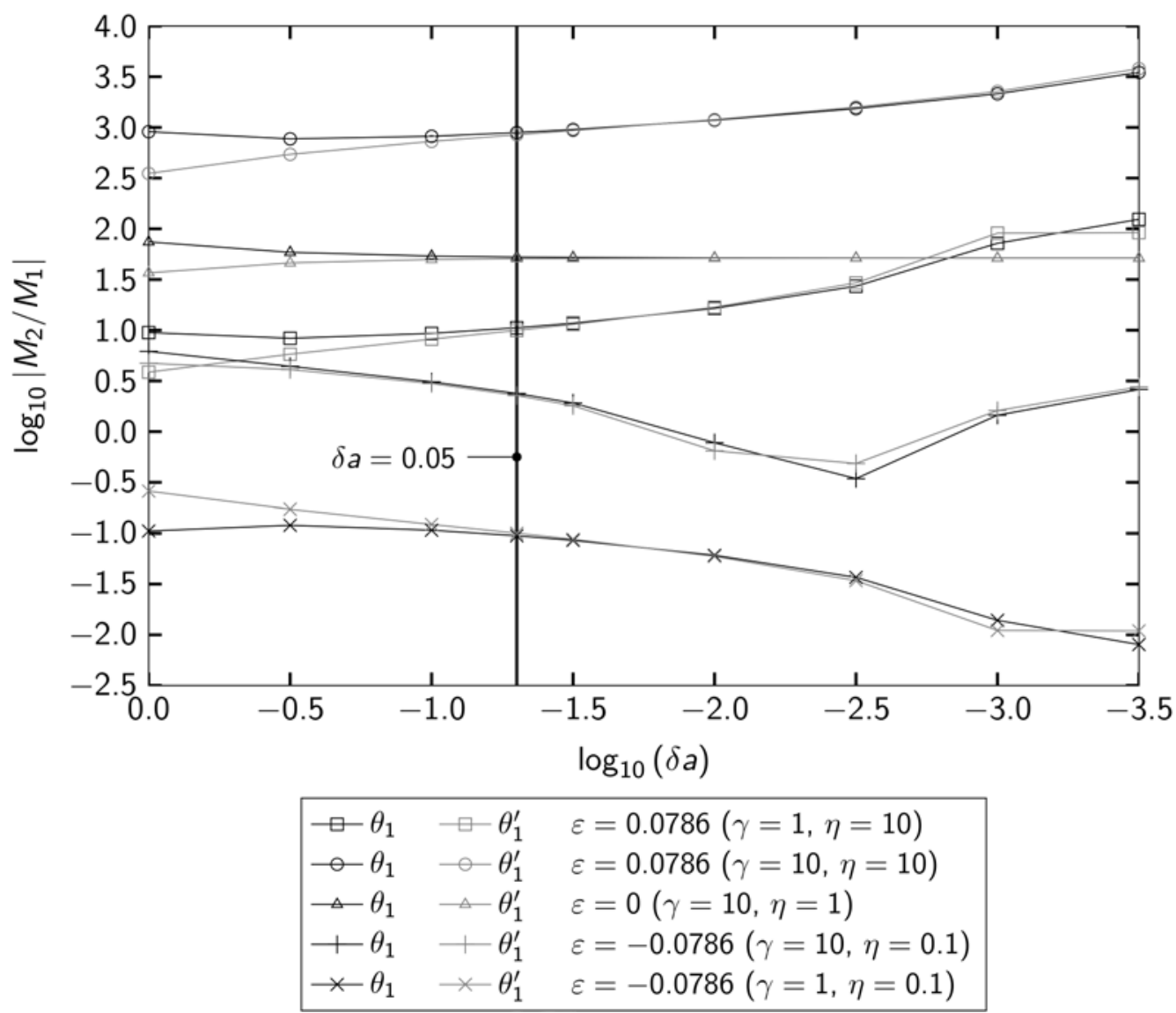

Fig. 3: Variation of $\theta_{1}$ and $\theta_{1}^{\prime}$ with respect to the crack extension size $\delta a$ for different values of the bimaterial mismatch constant $\varepsilon$ with $v_{1}=v_{2}=0.29$. 


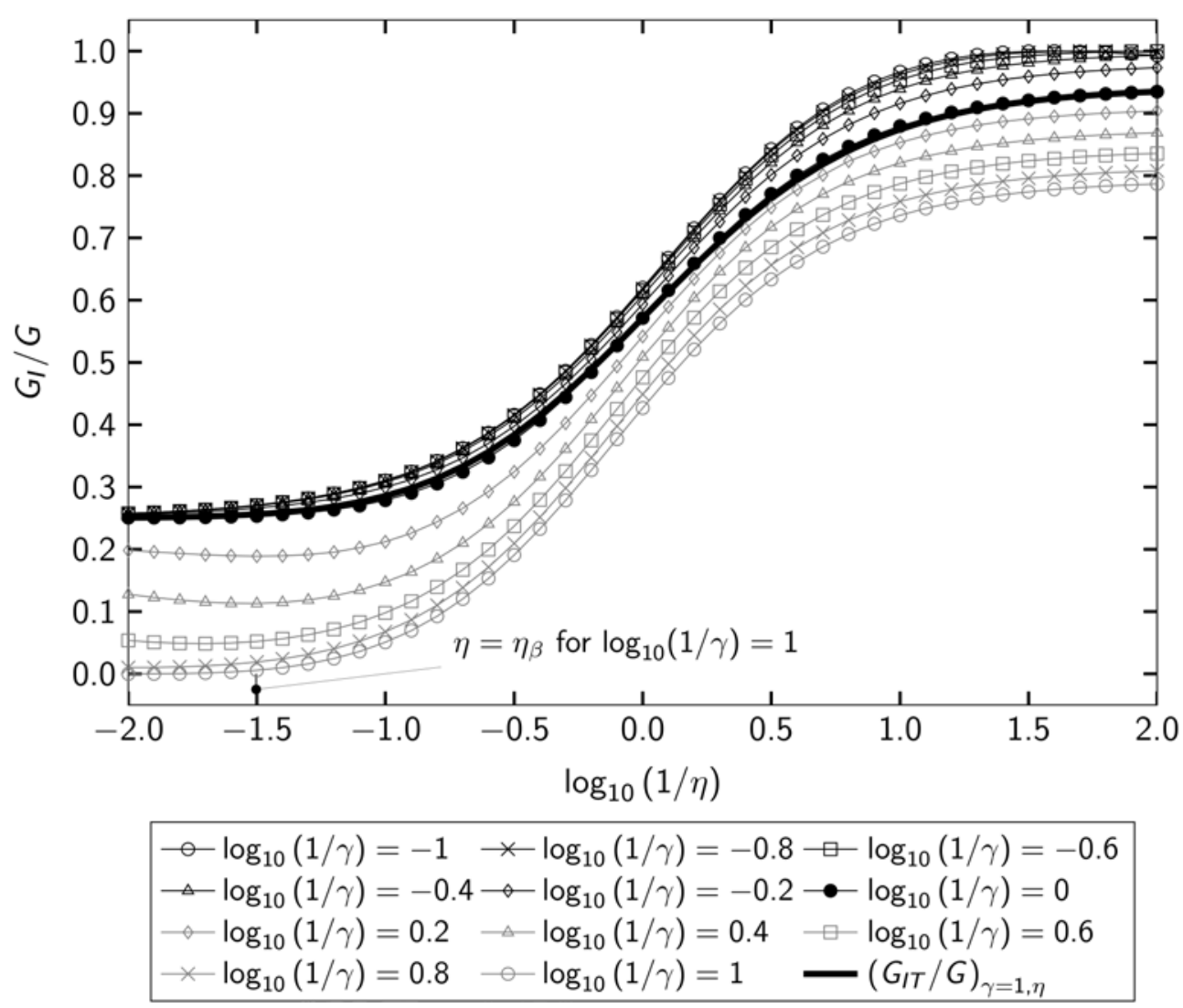

Fig. 4: FEM data for ERR partition $G_{I} / G$ based on the crack extension size $\delta a=0.05$ with $M_{2 B} / M_{1 B}=0$ and $v_{1}=v_{2}=0.29$. 